\title{
A Research on Toxicological Properties of Bursa City Parks's Design Plants
}

\author{
Betül Hümeyra ÇELIK ${ }^{1}$, Murat ZENCIRKIRAN ${ }^{2 *}$ \\ ${ }^{1}$ Bursa Uludağ University, Graduate School of Natural and Applied Science, Landscape Architecture Master's \\ Degree Program, Görükle Campus, Nilüfer - BURSA-TURKEY \\ ${ }^{2}$ Bursa Uludağ University, Faculty of Agriculture, Department of Landscape Architecture, Görükle Campus, \\ Nilüfer - BURSA-TURKEY
}

\begin{abstract}
The aim of this study is to reveal the toxicity of design (landscape) plants in Bursa city parks. Landscape plants used in design works are especially evaluated in terms of aesthetics and functionality. However, design (landscape) plants can pose a risk to human and animal health due to the toxic organic compounds they contain. Within this scope, the design (landscape) plants in Hudavendigar City Park, Soganli Botanical Park, Resat Oyal Culture Park and Merinos City Park, which are used extensively by the city's people and which are described as city parks, were examined in terms of their toxic properties. The results showed that the taxa included in the major toxicity group were found at the highest rate in Resat Oyal Culture Park, followed by Merinos City Park, Soganli Botanical Park and Hudavendigar City Park, respectively. Nerium oleander, Taxus baccata, Eriobotrya japonica, Sophora japonica, Pittosporum tobira can be given as examples of taxa included in the major toxicity group. It was determined that design (landscape) plants with major toxicity are used less in city parks, which have been designed and opened to service recently. In landscape design works, it is significant for designers know the negative effects that plants can have on humans and animals due to toxic components other than their esthetic and functional properties. In this way, designs will be made to account for the toxic compounds, toxin conditions of plants, the degree of toxin and the toxic organs of the plants, and the present toxicity will be the least dangerous.
\end{abstract}

Keywords: Bursa city parks, design (landscape) plants, poison classification, toxic compounds, toxic plants.

\section{Bursa Kent Parkları Tasarım Bitkilerinin Toksikolojik Özellikleri Üzerine Bir Araştırma}

\section{Öz}

$\mathrm{Bu}$ çalışmanın amacı, Bursa kent parklarında bulunan tasarım (peyzaj) bitkilerinin toksikolojik özelliklerini incelemek ve ortaya çıkarmaktır. Tasarım çalışmalarında kullanılan peyzaj bitkileri özellikle estetik ve işlevsellik açısından değerlendirilir. Ancak tasarım (peyzaj) bitkileri, içerdikleri toksik organik bileşikler nedeniyle insan ve hayvan sağlığı açısından risk oluşturabilir. Bu kapsamda şehir halkı tarafindan yoğun olarak kullanılan ve şehir parkı olarak nitelendirilen Hüdavendigar Kent Parkı, Soğanlı Botanik Parkı, Reşat Oyal Kültür Parkı ve Merinos Kent Parkı'nda bulunan tasarım (peyzaj) bitkileri, toksikolojik özellikleri açısından incelenmiştir. Sonuçlar, majör toksisite grubuna dahil olan taksonların en yüksek oranda Reşat Oyal Kültür Parkı'nda bulunduğunu, ardından sırasıyla Merinos Kent Parkı, Soğanlı Botanik Parkı ve Hüdavendigar Kent Parkı'nda bulunduğunu göstermiştir. Major toksisite grubuna dahil taksonlara Nerium oleander, Taxus baccata, Eriobotrya japonica, Sophora japonica, Pittosporum tobira örnek verilebilir. Yeni tasarlanan ve hizmete açılan şehir parklarında büyük toksisiteye sahip tasarım (peyzaj) bitkilerinin daha az kullanıldığı tespit edilmiştir. Peyzaj tasarım çalışmalarında, tasarımcıların bitkilerin estetik ve fonksiyonel özelliklerinin dışında bitkilerin sahip oldukları toksik bileşenlerden dolayı insanlar ve hayvanlar üzerinde oluşturabilecekleri olumsuz etkileri bilmesi önemlidir. Böylelikle bitkilerin toksik bileşiklerini, toksin durumlarını, toksin derecelerini ve bitkilerin toksik organlarını hesaba katacak tasarımlar yapılacak ve bitki toksisitesinden kaynaklanan tehlikeli durumlar en aza indirilecektir.

Anahtar Kelimeler: Bursa kent parkları, tasarım(peyzaj) bitkileri, zehir sınıflandırması, toksik bileşenler, toksik bitkiler.

\footnotetext{
*Sorumlu Yazar (Corresponding Author):

Murat ZENCIRKIRAN(Prof.); Bursa Uludag University, Faculty of Agriculture, 


\section{Introduction}

Design (landscape) plants, which are among the main elements of landscape architecture works, appear as live materials that complement each other in visual, functional and ecological terms. Design (landscape) plants are used for many purposes, such as screening of bad images, directing pedestrian traffic, preventing territorial erosion, psychological, emotional, mental and physical functions on humans (Scarfone 2007; Karasah and Var 2012; Sar1 and Karasah 2018; Akdeniz et al. 2017).

People primarily react to the external appearance of plants (Karasah and Var 2012), however, seeing the striking colors of flowers, picking fruits, smelling flowers and leaves of the plant, hearing the sounds that rain and wind make with plants are increase the quality of life immeasurable (Robinson 1992). At the same time, plants, which constitute a large part of the natural environment we live in and are assets that we need in many ways, have a positive or negative impact on human health and psychology (Lewis 1995; Frumkin 2001; Elings 2006; Martin et al. 2011; Zencirk1ran et al. 2018).

Many taxa used as indoor or outdoor design (landscape) plants may contain toxic substances that can cause harm effects on human and animal health (Lewis et al. 2007, Knight 2007, Filmer 2012). Toxic organic compounds (poison elements) present in plants are classified as essential oils, alkaloids, toxic amino acids, glycosides, resinous compounds, plant acids (oxalates), polyenes, tannins, proteins and peptides, saponins, terpenes and other toxic substances (Atasoy 2012). Contact with the sap of some plants, biting and ingestion of leaves, stems, fruits, seeds or roots can lead to emergence of the effects of toxic substances in the plants and may lead to much severe situations depending on the severity (Zencirkıran et al. 2018). The negative effects of toxic compounds on humans and animals can differ depending on the gender, age, body weight, health condition and immune system of organism (Muca et al. 2012).

The fact that people do not have enough information about the toxic effects of other plants except for toxic plants which are everyone knows, pose a risk at themselves and domestic animals. This situation makes significant to evaluate the plants we use in design works their toxic properties on humans and animals, as much as for their aesthetic and functional properties.

This study aims to reveal classifying the landscape plants used in the design of Bursa city parks according to their toxic properties and to raise awareness about the threats that may occur in terms of human and animal health.

\section{Material and Methods}

The province of Bursa is located in the southeast of Marmara Sea, between $40^{\circ}$ longitudes and $28^{\circ}-30^{\circ}$ latitudes. Hudavendigar City Park, Soganli Botanical Park, Resat Oyal Culture Park and Merinos City Park, which are in the status of city park in Bursa province, were selected as the research areas. Hudavendigar City Park, located in the northwest of Bursa province with an area of 510,000 square meter, is the largest city park in Bursa province and was opened in 2015. Soganlı Botanical Park; located in the north of Bursa province, the city park, with a large area of 400,000 square meter, was opened in 1998. The Resat Oyal Cultur Park, located close to the city center of Bursa, is the city park with a size of 400,000 square meter and was opened in 1955. Merinos City Park, located close to the city center of Bursa, is the city park with a size of 252,500 square meter and was opened in 2008 (Figure 1).

The materials of the study are woody and herbaceous design (landscape) plants used in the landscape design of four city parks selected as research areas. 



Figure 1.The location of Bursa province and the study areas (Zencirkiran et.al. 2019)

The study was carried out in three phases. In the first phase, city parks were visited in two different periods (spring and autumn) in 2019, in order to identify woody and herbaceous design (landscape) plants. During these visits, plant samples were taken, photographs of taxa were taken and the taxa were diagnosed using Davis (1965-1988), Polunin (1969), Polunin and Huxley (1981), Krusmann (1984-1986), Dirr (1992), Yaltirik (1993), Anonymous (1998), Zencirkiran (2004), Zencirkiran (2009), Zencirkiran (2013).

In the second phase, toxic properties of taxa detected in city parks were evaluated. To create toxicity groups have been benefited from Filmer (2012), Atasoy (2012) and Zencirkiran et al. (2018). And six (6) groups were formed which given below. It was identified in which group the taxa are located.

Group 1. Major Toxicity: These plants may cause serious illness or death.

Group 2. Minor Toxicity: Ingestion of these plants may cause minor illnesses such as vomiting or diarrhea.

Group 3. Oxalates: The juice or sap of these plants contains oxalate crystals. These needle-shaped crystals can irritate the skin, mouth, tongue, and throat, resulting in throat swelling, breathing difficulties, burning pain, and stomach upset.

Group 4. Dermatitis: The juice, sap, or thorns of these plants may cause a skin rash or irritation.

Group 5. Animal toxicity: Plants in this group are poisonous to animals such as cats and dogs.

Group 6. Non-toxic: Plants in this group are not harmed.

It was determined in which toxic groups the taxa were included using Baytop (1963), Y1lmaz (1990), Altıntaş (1995), Yilmaz et al. (2006), Knight (2007), Lewis et al. (2007), Wagstaff (2008), Aydın (2010), Yener and Seyidoglu (2010), Filmer (2012), Di Tomaso (2019).

All data obtained were evaluated using the SPSS 22 for Windows package program, by means covering the city parks as a whole and individually, in the third phase (IBM Corp. Released 2013). The correlation analyzes were done to determine the relationship between the total number of taxa and toxic taxa and between the number of taxa and toxic groups. The One-Way Anova test was used in the evaluation of city parks, and the Two-Way Anova test was used in the evaluation of taxa and toxic groups being in city parks. Separation of means was done by Duncan multiple range tests. 


\section{Results}

\subsection{The distribution of taxa by city parks and toxic groups}

The distributions and toxic groups of woody and herbaceous taxa found in city parks are given in Table 1 . The mostly woody and herbaceous taxa were determined in Soganli Botanical Park with 159, followed by Hüdavendigar City Park and Resat Oyal Culture Park with 108 and 105 taxa, respectively, and the lowest taxa were determined in Merinos City Park with 78 taxa.

Table 1.The distribution of woody and herbaceous taxa in city parks and their toxic groups

\begin{tabular}{|c|c|c|c|c|c|}
\hline Taxons & City Park* & $\begin{array}{l}\text { Toxic } \\
\text { Groups }\end{array}$ & Taxon & City Park* & $\begin{array}{l}\text { Toxic } \\
\text { Groups }\end{array}$ \\
\hline Acer campestre L. & $\mathrm{B}$ & 6 & $\begin{array}{l}\text { Mesembryanthemum } \\
\text { cooperi Hook.f. }\end{array}$ & $\mathrm{A}$ & 6 \\
\hline Acer ginnala Maxim. & B & 6 & $\begin{array}{l}\text { Cotinus coggygria } \\
\text { Scop. }\end{array}$ & A,B & 4 \\
\hline Acer negundo L. & $\mathrm{B}, \mathrm{C}$ & 6 & $\begin{array}{l}\text { Rhus typhina L. } \\
\text { 'Dissecta' }\end{array}$ & $\mathrm{B}$ & 6 \\
\hline $\begin{array}{l}\text { Acer negundo L. } \\
\text { 'Flamingo' }\end{array}$ & $\mathrm{B}$ & 6 & Nerium oleander L. & $\mathrm{A}, \mathrm{B}, \mathrm{D}$ & $1,4,5$ \\
\hline $\begin{array}{l}\text { Acer negundo L. } \\
\text { 'Variegatum' }\end{array}$ & $\mathrm{B}$ & 6 & Ilex aquifolium $\mathrm{L}$. & $\mathrm{B}$ & 2,5 \\
\hline $\begin{array}{l}\text { Acer palmatum Thunb. } \\
\text { 'Atropurpureum' }\end{array}$ & B & 6 & $\begin{array}{l}\text { Ilex aquifolium } \\
\text { L.'Argentea } \\
\text { Marginata' }\end{array}$ & A & 2,5 \\
\hline $\begin{array}{l}\text { Acer palmatum } \\
\text { Thunb. 'Dissectum } \\
\text { Atropurpureum' }\end{array}$ & $\mathrm{B}, \mathrm{C}$ & 6 & $\begin{array}{l}\text { Ilex aquifolium } \\
\text { L.'Variegata' }\end{array}$ & B & 2,5 \\
\hline $\begin{array}{l}\text { Acer palmatum } \\
\text { Thunb. 'Dissectum Garnet' }\end{array}$ & $\mathrm{A}, \mathrm{B}$ & 6 & $\begin{array}{l}\text { Ilex crenata } \\
\text { Thunb.'Kinme' }\end{array}$ & A & 2,5 \\
\hline $\begin{array}{l}\text { Acer palmatum } \\
\text { Thunb.'Dissectum Viridis' }\end{array}$ & $\mathrm{B}$ & 6 & Colocasia sp. & $\mathrm{D}$ & 2,5 \\
\hline Acer platanoides L. & $\mathrm{B}, \mathrm{C}, \mathrm{D}$ & 6 & Hedera helix L. & $\mathrm{C}$ & $1,2,4,5$ \\
\hline $\begin{array}{l}\text { Acer platanoides } \\
\text { L.'Crimson King' }\end{array}$ & A,B & 6 & $\begin{array}{l}\text { Washingtonia robusta } \\
\text { H.Wendl. }\end{array}$ & $\mathrm{B}$ & 6 \\
\hline $\begin{array}{l}\text { Acer platanoides L. } \\
\text { 'Deborah' }\end{array}$ & B & 6 & $\begin{array}{l}\text { Chamaerops excelsa } \\
\text { Thunb. }\end{array}$ & $\mathrm{C}$ & 6 \\
\hline $\begin{array}{l}\text { Acer platanoides } \\
\text { L.'Globosum' }\end{array}$ & $\mathrm{B}$ & 6 & $\begin{array}{l}\text { Chamaerops humilis } \\
\text { L. }\end{array}$ & $\mathrm{C}$ & 6 \\
\hline Acer pseudoplatanus L. & $\mathrm{B}, \mathrm{C}$ & 4,5 & $\begin{array}{l}\text { Ageratum } \\
\text { houstonianum Mill. }\end{array}$ & A,B,D & 6 \\
\hline Acer rubrum $\mathrm{L}$. & $\mathrm{B}$ & 4,5 & $\begin{array}{l}\text { Aster dumosus } \\
\text { Hoffm. 'Schneekissen' }\end{array}$ & A & 6 \\
\hline Acer saccharinum $\mathrm{L}$. & A,D & 4,5 & $\begin{array}{l}\text { Aster dumosus } \\
\text { Hoffm.' 'Wood's } \\
\text { Pink' }\end{array}$ & A & 6 \\
\hline Acer saccharum Marshall & B & 4,5 & Dahlia sp. & $\mathrm{A}, \mathrm{B}, \mathrm{C}$ & 6 \\
\hline Agave americana $\mathrm{L}$. & $\mathrm{B}$ & $2,3,4,5$ & $\begin{array}{l}\text { Euryops pectinatus } \\
\text { (L.) Cass. }\end{array}$ & A,C,D & 6 \\
\hline $\begin{array}{l}\text { Phormium tenax J.R.Forst. } \\
\text { \&G.Forst. }\end{array}$ & $\mathrm{D}$ & 6 & Gazania sp. & $\mathrm{A}, \mathrm{C}$ & 6 \\
\hline $\begin{array}{l}\text { Phormium tenax J.R.Forst. } \\
\text { \&G.Forst. 'Variegata' }\end{array}$ & $\mathrm{B}, \mathrm{D}$ & 6 & $\begin{array}{l}\text { Liatris spicata }(\mathrm{L} .) \\
\text { Willd. }\end{array}$ & A & 6 \\
\hline Yucca filamentosa $\mathrm{L}$. & $\mathrm{B}, \mathrm{C}$ & 5 & $\begin{array}{l}\text { Senecio greyii Parry } \\
\text { ex A.Gray }\end{array}$ & A & $1,2,4,5$ \\
\hline
\end{tabular}

*City Park: A-Hüdavendigar, B-Soganli Botanical, C-Resat Oyal, D-Merinos 
Table 1. Continuing

\begin{tabular}{|c|c|c|c|c|c|}
\hline Taxons & City Park* & $\begin{array}{l}\text { Toxic } \\
\text { Groups }\end{array}$ & Taxon & $\begin{array}{l}\text { City } \\
\text { Park* }\end{array}$ & $\begin{array}{l}\text { Toxic } \\
\text { Groups }\end{array}$ \\
\hline $\begin{array}{l}\text { Tanacetum parthenium } \\
\text { (L.) Sch. Bip. }\end{array}$ & B & 4 & $\begin{array}{l}\text { Catalpa bignonoides } \\
\text { Walter }\end{array}$ & $\mathrm{B}, \mathrm{C}$ & 6 \\
\hline Tagetes erecta $\mathrm{L}$. & A,B,C,D & 4 & $\begin{array}{l}\text { Alyssum saxatile } \\
\text { L. 'Compactum' }\end{array}$ & A & 6 \\
\hline Impatiens sp. & C,D & 6 & $\begin{array}{l}\text { Brassica oleraceae var. } \\
\text { acephala DC. }\end{array}$ & $\mathrm{B}$ & 6 \\
\hline $\begin{array}{l}\text { Impatiens walleriana } \\
\text { Hook.f. }\end{array}$ & B & 6 & Budleja davidii Franch. & $\mathrm{B}, \mathrm{D}$ & 6 \\
\hline Begonia sp. & C,D & $2,3,5$ & $\begin{array}{l}\text { Buxus microphylla } \\
\text { Siebold. \& Zucc. }\end{array}$ & $\mathrm{C}$ & 6 \\
\hline $\begin{array}{l}\text { Berberis juliana } \\
\text { C.K.Schneid. }\end{array}$ & B & 2,4 & $\begin{array}{l}\text { Buxus microphylla } \\
\text { Siebold. \& Zucc. } \\
\text { 'Faulkner' }\end{array}$ & $\mathrm{A}, \mathrm{C}$ & 6 \\
\hline $\begin{array}{l}\text { Berberis thunbergii DC. } \\
\text { 'Atropurpurea' }\end{array}$ & C,D & 2,4 & $\begin{array}{l}\text { Buxus macrophylla } \\
\text { Fawc. \& Rendle }\end{array}$ & B & 6 \\
\hline $\begin{array}{l}\text { Berberis thunbergii } \\
\text { DC.'Maria' }\end{array}$ & A & 2,4 & Buxus sempervirens L. & $\mathrm{B}, \mathrm{C}, \mathrm{D}$ & $2,4,5$ \\
\hline $\begin{array}{l}\text { Berberis thunbergii DC. } \\
\text { 'Orange Rocket' }\end{array}$ & A & 2,4 & $\begin{array}{l}\text { Buxus sempervirens L. } \\
\text { 'Suffruticosa' }\end{array}$ & A & $2,4,5$ \\
\hline $\begin{array}{l}\text { Berberis thunbergii DC. } \\
\text { 'Tiny Gold' }\end{array}$ & A & 2,4 & Canna indica $\mathrm{L}$. & $\mathrm{C}$ & 6 \\
\hline $\begin{array}{l}\text { Nandina domestica } \\
\text { Thunb. }\end{array}$ & $\mathrm{C}$ & 5 & $\begin{array}{l}\text { Abelia } x \text { grandiflora } \\
\text { (Rovelli ex Andre) } \\
\text { Rehder }\end{array}$ & A,B,C,D & 6 \\
\hline $\begin{array}{l}\text { Nandina domestica } \\
\text { Thunb. 'Firepower' }\end{array}$ & A,D & 5 & Lonicera sp. & $\mathrm{C}$ & 2,5 \\
\hline $\begin{array}{l}\text { Alnus glutinosa ( L.) } \\
\text { Gaertn. }\end{array}$ & B & 4 & Sambucus nigra L. & $\mathrm{C}$ & 1,5 \\
\hline Betula pendula Roth. & $\mathrm{A}, \mathrm{B}, \mathrm{C}$ & 2,4 & Viburnum lucidum Mill. & $\mathrm{C}$ & 6 \\
\hline $\begin{array}{l}\text { Betula pendula } \\
\text { Roth.'Youngii' }\end{array}$ & A & 2,4 & Viburnum opulus L. & $\mathrm{B}, \mathrm{D}$ & 6 \\
\hline $\begin{array}{l}\text { Betula verrucosa } \\
\text { Ehrh.'Youngii' }\end{array}$ & B & 6 & Viburnum tinus L. & $\mathrm{B}, \mathrm{D}$ & 6 \\
\hline Carpinus betulus L. & B & 6 & $\begin{array}{l}\text { Weigela florida Bunge. } \\
\text { A. DC. }\end{array}$ & A & 6 \\
\hline $\begin{array}{l}\text { Carpinus betulus } \\
\text { L.'Pendula' }\end{array}$ & $\mathrm{B}$ & 6 & $\begin{array}{l}\text { Euonymus alatus } \\
\text { (Thunb.) Siebold }\end{array}$ & $\mathrm{B}$ & 2 \\
\hline Corylus avellana $\mathrm{L}$. & $\mathrm{C}$ & 2,4 & $\begin{array}{l}\text { Euonymus japonicus } \\
\text { Thunb.'Aurea' }\end{array}$ & $\mathrm{B}, \mathrm{C}$ & 2 \\
\hline Corylus maxima Mill. & B & 6 & $\begin{array}{l}\text { Euonymus japonicus } \\
\text { Thunb.'Elegantissima } \\
\text { Aurea' }\end{array}$ & $\mathrm{A}, \mathrm{C}$ & 2 \\
\hline $\begin{array}{l}\text { Campsis radicans }(\mathrm{L} .) \\
\text { Seem. }\end{array}$ & B & 4 & $\begin{array}{l}\text { Euonymus japonicus } \\
\text { Thunb. 'Pflege' }\end{array}$ & $\mathrm{C}$ & 2 \\
\hline $\begin{array}{l}\text { Euonymus japonicus } \\
\text { Thunb. 'Pulchellus } \\
\text { Aureovariegatus' }\end{array}$ & A & 2 & $\begin{array}{l}\text { Cupressus macrocarpa } \\
\text { Gord. 'Goldcrest' }\end{array}$ & B & 6 \\
\hline $\begin{array}{l}\text { Euonymus japonicus } \\
\text { Thunb.'Variegata' }\end{array}$ & $\mathrm{D}$ & 2 & $\begin{array}{l}\text { Cupressus sempervirens } \\
\text { L. }\end{array}$ & $\mathrm{B}, \mathrm{C}, \mathrm{D}$ & 6 \\
\hline
\end{tabular}

*City Park: A-Hüdavendigar, B-Soganli Botanical, C-Resat Oyal, D-Merinos 
Table 1. Continuing

\begin{tabular}{|c|c|c|c|c|c|}
\hline Taxons & City Park* & $\begin{array}{l}\text { Toxic } \\
\text { Groups }\end{array}$ & Taxon & $\begin{array}{l}\text { City } \\
\text { Park* }\end{array}$ & $\begin{array}{l}\text { Toxic } \\
\text { Groups }\end{array}$ \\
\hline Convolvulus arvensis $\mathrm{L}$. & $\mathrm{A}, \mathrm{B}, \mathrm{C}, \mathrm{D}$ & 5 & $\begin{array}{l}\text { Cupressus sempervirens } \\
\text { L.'Pyramidalis' }\end{array}$ & $\mathrm{A}$ & 6 \\
\hline $\begin{array}{l}\text { Cornus florida f. rubra } \\
\text { (Weston) E.J.Palmer \& } \\
\text { Steyerm., }\end{array}$ & $\mathrm{D}$ & 6 & $\begin{array}{l}\text { Juniperus conferta Parl.'Blue } \\
\text { Pacific' }\end{array}$ & A & 6 \\
\hline $\begin{array}{l}\text { Sempervivum } \\
\text { arachnoideum } \mathrm{L} \text {. }\end{array}$ & A & 6 & $\begin{array}{l}\text { Juniperus x pfitzeriana } \\
\text { (Späth) P.A.Schmidt 'Aurea' }\end{array}$ & $\mathrm{D}$ & 6 \\
\hline $\begin{array}{l}\text { Sempervivum ciliosum } \\
\text { 'Borisii' }\end{array}$ & A & 6 & $\begin{array}{l}\text { Juniperus x pfitzeriana } \\
\text { (Späth) P.A.Schmidt } \\
\text { 'Glauca' }\end{array}$ & $\mathrm{D}$ & 6 \\
\hline $\begin{array}{l}\text { Sempervivum tectorum } \\
\text { L. }\end{array}$ & A & 6 & $\begin{array}{l}\text { Juniperus sabina } \\
\text { L.'Tamariscifolia' }\end{array}$ & $\mathrm{D}$ & $1,2,5$ \\
\hline $\begin{array}{l}\text { Calocedrus decurrens } \\
\text { (Torr.) Florin }\end{array}$ & $\mathrm{A}, \mathrm{B}$ & 6 & $\begin{array}{l}\text { Juniperus squamata Buch.- } \\
\text { Ham. Ex Lamb.'Blue Star’ }\end{array}$ & A & 6 \\
\hline $\begin{array}{l}\text { Calocedrus decurrens } \\
\text { (Torr.) Florin } \\
\text { 'Aureovariegata' }\end{array}$ & A & 6 & $\begin{array}{l}\text { Juniperus squamata Buch.- } \\
\text { Ham. Ex Lamb. 'Gold Star' }\end{array}$ & A & 6 \\
\hline $\begin{array}{l}\text { Chamaecyparis } \\
\text { lawsoniana (Murr.) } \\
\text { Parl.'Elwoodi’ }\end{array}$ & $\mathrm{A}, \mathrm{B}$ & 6 & $\begin{array}{l}\text { Juniperus virginiana } \\
\text { L.'Skyrocket' }\end{array}$ & B & $2,4,5$ \\
\hline $\begin{array}{l}\text { Chamaecyparis } \\
\text { lawsoniana (Murr.) } \\
\text { Parl.' 'Stardust' }\end{array}$ & B & 6 & $\begin{array}{l}\text { Metasequoia } \\
\text { glyptostroboides Hu \& } \\
\text { W.C.Cheng }\end{array}$ & $\mathrm{B}$ & 6 \\
\hline $\begin{array}{l}\text { Chamaecyparis } \\
\text { nootkatensis (D.Don) } \\
\text { Spach 'Pendula' }\end{array}$ & B & 6 & $\begin{array}{l}\text { Platycladus orientalis (L.) } \\
\text { Franco }\end{array}$ & $\mathrm{C}$ & $2,4,5$ \\
\hline $\begin{array}{l}\text { Chamaecyparis obtusa } \\
\text { (Siebold \&Zucc.) } \\
\text { Endl.'Nana Gracilis' }\end{array}$ & B & 6 & $\begin{array}{l}\text { Platycladus orientalis (L.) } \\
\text { Franco 'Compacta Nana' }\end{array}$ & B & $2,4,5$ \\
\hline $\begin{array}{l}\text { Chamaecyparis pisifera } \\
\text { (Siebold \& Zucc.) Endl. }\end{array}$ & B & 6 & $\begin{array}{l}\text { Platycladus orientalis (L.) } \\
\text { Franco 'Compacta Aurea } \\
\text { Nana' }\end{array}$ & B & $2,4,5$ \\
\hline $\begin{array}{l}\text { Cryptomeria japonica } \\
\text { (Thunb. ex L.f.) D.Don }\end{array}$ & $\mathrm{B}, \mathrm{C}$ & 6 & $\begin{array}{l}\text { Platycladus orientalis (L.) } \\
\text { Franco 'Golden Anne' }\end{array}$ & A & $2,4,5$ \\
\hline $\begin{array}{l}\text { Cryptomeria japonica } \\
\text { (Thunb. ex L.f.) D.Don } \\
\text { 'Globosa Nana' }\end{array}$ & B & 6 & Thuja occidentalis L. & $\mathrm{C}$ & 2,4 \\
\hline $\begin{array}{l}\text { X Cupressocyparis } \\
\text { leylandii (A.B.Jacks. \& } \\
\text { Dallim.) Dallim. }\end{array}$ & $\mathrm{A}, \mathrm{B}$ & 6 & $\begin{array}{l}\text { Thuja occidentalis } \\
\text { L.'Forever Gold' }\end{array}$ & A & 2,4 \\
\hline $\begin{array}{l}\text { X Cupressocyparis } \\
\text { leylandii (A.B.Jacks. \& } \\
\text { Dallim.)Dallim. 'Cast. } \\
\text { Gold' }\end{array}$ & B & 6 & $\begin{array}{l}\text { Thuja occidentalis L. 'Golden } \\
\text { Toffet' }\end{array}$ & A & 2,4 \\
\hline $\begin{array}{l}\text { Cupressus arizonica } \\
\text { Greene }\end{array}$ & C & 6 & $\begin{array}{l}\text { Thuja occidentalis } \\
\text { L.'Mirjam' }\end{array}$ & A & 2,4 \\
\hline $\begin{array}{l}\text { Cupressus arizonica } \\
\text { Greene 'Glauca' }\end{array}$ & $\mathrm{B}, \mathrm{D}$ & 6 & $\begin{array}{l}\text { Thuja occidentalis } \\
\text { L.'Emerald' }\end{array}$ & B & 2,4 \\
\hline $\begin{array}{l}\text { Cupressus macrocarpa } \\
\text { Gord. 'Aurea' }\end{array}$ & B & 6 & $\begin{array}{l}\text { Thuja occidentalis } \\
\text { L.'Pyramidalis' }\end{array}$ & B & 2,4 \\
\hline
\end{tabular}

*City Park: A-Hüdavendigar, B-Soganli Botanical, C-Resat Oyal, D-Merinos 
Table 1. Continuing

\begin{tabular}{|c|c|c|c|c|c|}
\hline Taxons & City Park* & $\begin{array}{l}\text { Toxic } \\
\text { Groups }\end{array}$ & Taxon & $\begin{array}{l}\text { City } \\
\text { Park* }\end{array}$ & $\begin{array}{l}\text { Toxic } \\
\text { Groups }\end{array}$ \\
\hline $\begin{array}{l}\text { Thuja occidentalis } \\
\text { L.'Rheingold' }\end{array}$ & B & 2,4 & $\begin{array}{l}\text { Wisteria sinensis (Sims) } \\
\text { Sweet }\end{array}$ & $\mathrm{B}, \mathrm{C}, \mathrm{D}$ & 2,5 \\
\hline $\begin{array}{l}\text { Thuja plicata Donn ex } \\
\text { D.Don }\end{array}$ & A,C,D & 2,4 & Fagus sylvatica $\mathrm{L}$. & B & 6 \\
\hline $\begin{array}{l}\text { Thuja plicata Donn ex } \\
\text { D.Don 'Atrovirens' }\end{array}$ & B & 2,4 & $\begin{array}{l}\text { Fagus sylvatica } \\
\text { L.'Atropurpurea' }\end{array}$ & A & 6 \\
\hline Carex oshimensis Nakai & B & 6 & Fagus sylvatica L.'Tricolor' & A & 6 \\
\hline $\begin{array}{l}\text { Carex oshimensis Nakai } \\
\text { 'Evergold' }\end{array}$ & A & 6 & Quercus ilex $\mathrm{L}$. & $\mathrm{A}, \mathrm{B}$ & $2,4,5$ \\
\hline Diospyros kaki L.f. & $\mathrm{C}$ & 6 & Quercus palustris Münchh. & B & 2,5 \\
\hline Diospyros lotus L. & $\mathrm{C}$ & 6 & Quercus robur $\mathrm{L}$. & $\mathrm{A}, \mathrm{C}$ & 2,5 \\
\hline $\begin{array}{l}\text { Elaeagnus angustifolia } \\
\text { L. }\end{array}$ & $\mathrm{B}, \mathrm{C}$ & 6 & Quercus robur L.'Fastigiata' & B & 2,5 \\
\hline $\begin{array}{l}\text { Elaeagnus pungens } \\
\text { Thunb. }\end{array}$ & A,C,D & 6 & Quercus rubra $\mathrm{L}$. & $\mathrm{B}, \mathrm{D}$ & 2,5 \\
\hline Arbutus unedo $\mathrm{L}$. & A & 6 & $\begin{array}{l}\text { Quercus rubra } \\
\text { L.'Americana' }\end{array}$ & $\mathrm{B}$ & 2,5 \\
\hline Rhododendron. sp. L. & $\mathrm{B}, \mathrm{D}$ & 1,5 & $\begin{array}{l}\text { Quercus rubra } \\
\text { L.'Pyramidalis' }\end{array}$ & B & 2,5 \\
\hline Acacia dealbata Link & $\mathrm{C}$ & 6 & Pelargonium sp.L.'Her.. & $\mathrm{B}, \mathrm{D}$ & 6 \\
\hline $\begin{array}{l}\text { Albizzia julibrissin } \\
\text { Durazz. }\end{array}$ & A,B,C,D & 5 & $\begin{array}{l}\text { Pelargonium peltatum (L.) } \\
\text { L'Hér. }\end{array}$ & A & 6 \\
\hline Cercis siliquastrum $\mathrm{L}$. & $\mathrm{A}, \mathrm{B}, \mathrm{C}, \mathrm{D}$ & 6 & Ginkgo biloba $\mathrm{L}$. & $\mathrm{A}, \mathrm{B}, \mathrm{C}$ & 2,4 \\
\hline Gleditsia triacanthos $\mathrm{L}$. & $\mathrm{C}$ & 6 & Liquidambar styraciflua $\mathrm{L}$. & A,B,D & 6 \\
\hline $\begin{array}{l}\text { Gleditsia triacanthos } \\
\text { L.'Sunburst' }\end{array}$ & A & 6 & Aesculus x carnea Hayne & C,D & 2,5 \\
\hline $\begin{array}{l}\text { Laburnum watereri } \\
\text { (Wettst.) Dippel }\end{array}$ & B & 2,5 & Aesculus hippocastanum L. & $\mathrm{B}, \mathrm{C}$ & 2,5 \\
\hline $\begin{array}{l}\text { Robinia pseudoacacia } \\
\text { L. }\end{array}$ & $\mathrm{B}, \mathrm{C}$ & 1,5 & $\begin{array}{l}\text { Hydrangea macrophylla } \\
\text { (Thunb.) Ser. }\end{array}$ & $\mathrm{A}, \mathrm{C}$ & $1,4,5$ \\
\hline $\begin{array}{l}\text { Robinia pseudoacacia } \\
\text { L.'Umbraculifera' }\end{array}$ & B & 1,5 & Juglans nigra $\mathrm{L}$. & B & 4,5 \\
\hline Sophora japonica L. & $\mathrm{B}, \mathrm{C}$ & 1,2 & Juglans regia L. & $\mathrm{C}$ & 4,5 \\
\hline $\begin{array}{l}\text { Sophora japonica } \\
\text { L.'Pendula' }\end{array}$ & $\mathrm{A}$ & 1,2 & $\begin{array}{l}\text { Salvia splendens Sellow ex } \\
\text { Schult. }\end{array}$ & $\mathrm{A}, \mathrm{B}, \mathrm{C}$ & 6 \\
\hline Laurus nobilis L. & $\mathrm{C}, \mathrm{B}$ & 4 & Mirabilis jalapa $\mathrm{L}$. & $\mathrm{D}$ & $2,4,5$ \\
\hline Hemerocallis fulva $\mathrm{L}$. & A & 6 & Fraxinus angustifolia Vahl. & $\mathrm{C}$ & 6 \\
\hline Kniphofia sp. & A & 6 & $\begin{array}{l}\text { Fraxinus angustifolia } \\
\text { Vahl.'Raywood' }\end{array}$ & A & 6 \\
\hline $\begin{array}{l}\text { Ophiopogon } \\
\text { planiscapus Nakai } \\
\text { 'Nigrescens' }\end{array}$ & A & 6 & Fraxinus excelsior L. & $\mathrm{C}$ & 6 \\
\hline Lagerstroemia indica $\mathrm{L}$. & A.B,C,D & 6 & $\begin{array}{l}\text { Fraxinus excelsior } \\
\text { L.'Pendula' }\end{array}$ & B & 6 \\
\hline $\begin{array}{l}\text { Liriodendron tulipifera } \\
\text { L. }\end{array}$ & $\mathrm{B}, \mathrm{C}, \mathrm{D}$ & 6 & Forsythia $\mathrm{x}$ intermedia Zabel & $\mathrm{D}$ & 6 \\
\hline $\begin{array}{l}\text { Magnolia grandiflora } \\
\text { L. }\end{array}$ & A,B,C,D & 6 & Jasminum nudiflorum Lindl. & $\mathrm{B}, \mathrm{D}$ & 6 \\
\hline $\begin{array}{l}\text { Magnolia liliiflora } \\
\text { Desr. }\end{array}$ & A & 6 & Ligustrum ionandrum Diels & $\mathrm{A}, \mathrm{B}$ & $2,4,5$ \\
\hline
\end{tabular}

*City Park: A-Hüdavendigar, B-Soganli Botanical, C-Resat Oyal, D-Merinos 
Table 1. Continuing

\begin{tabular}{|c|c|c|c|c|c|}
\hline Taxons & City Park* & $\begin{array}{l}\text { Toxic } \\
\text { Groups }\end{array}$ & Taxon & $\begin{array}{l}\text { City } \\
\text { Park* }\end{array}$ & $\begin{array}{l}\text { Toxic } \\
\text { Groups }\end{array}$ \\
\hline $\begin{array}{l}\text { Magnolia } \mathrm{x} \\
\text { soulangeana Soul.-Bod. }\end{array}$ & $\mathrm{B}, \mathrm{C}, \mathrm{D}$ & 6 & Ligustrum ovalifolium Hassk. & A & $2,4,5$ \\
\hline $\begin{array}{l}\text { Magnolia stellata } \\
\text { (Siebold \& Zucc.) } \\
\text { Maxim. }\end{array}$ & $\mathrm{B}$ & 6 & Ligustrum vulgare L. & C,D & $2,4,5$ \\
\hline Alcea rosea $\mathrm{L}$. & $\mathrm{C}$ & 4,5 & Olea europaea L. & $\mathrm{C}$ & 6 \\
\hline Hibiscus syriacus L. & $\mathrm{A}, \mathrm{B}, \mathrm{C}$ & 5 & $\begin{array}{l}\text { Osmanthus aquifolium } \\
\text { Siebold ex Siebold \& Zucc. }\end{array}$ & $\mathrm{B}$ & 6 \\
\hline Tilia argentea DC. & $\mathrm{C}$ & 6 & Syringa vulgaris $\mathrm{L}$. & $\mathrm{B}, \mathrm{D}$ & 6 \\
\hline Tilia tomentosa Moench & A,B,D & 6 & $\begin{array}{l}\text { Oenothera lindheimeri } \\
\text { (Engelm. \& A. Gray) W.L. } \\
\text { Wagner \& Hoch }\end{array}$ & A & 6 \\
\hline Melia azedarach L. & $\mathrm{C}$ & 1,5 & $\begin{array}{l}\text { Pittosporum tobira (Thunb.) } \\
\text { W.T.Aiton }\end{array}$ & $\mathrm{C}$ & 1,5 \\
\hline $\begin{array}{l}\text { Broussonetia papyrifera } \\
\text { (L.) Vent. }\end{array}$ & $\mathrm{C}$ & 6 & $\begin{array}{l}\text { Pittosporum tobira (Thunb.) } \\
\text { W.T.Aiton 'Nana' }\end{array}$ & B & 1,5 \\
\hline Ficus carica $\mathrm{L}$. & $\mathrm{C}$ & 4 & $\begin{array}{l}\text { Platanus x acerifolia (Aiton) } \\
\text { Willd. }\end{array}$ & B & 6 \\
\hline Morus alba L. & $\mathrm{B}, \mathrm{C}$ & 6 & Platanus orientalis L. & $\mathrm{A}, \mathrm{C}$ & 6 \\
\hline Morus alba L.'Pendula' & $\mathrm{B}$ & 6 & Abies alba Mill. & $\mathrm{B}, \mathrm{C}$ & 6 \\
\hline Morus nigra L. & $\mathrm{C}$ & 6 & $\begin{array}{l}\text { Abies bornmuelleriana } \\
\text { Mattf. }\end{array}$ & $\mathrm{B}, \mathrm{C}, \mathrm{D}$ & 6 \\
\hline Callistemon sp. & $\mathrm{D}$ & 4 & $\begin{array}{l}\text { Abies concolor (Gordon \& } \\
\text { Glend) Lindl. ex Hildebr. }\end{array}$ & A & 6 \\
\hline $\begin{array}{l}\text { Abies nordmanniana } \\
\text { (Steven) Spach }\end{array}$ & $\mathrm{A}, \mathrm{B}$ & 6 & $\begin{array}{l}\text { Picea pungens Engelm. } \\
\text { 'Hoopsi' }\end{array}$ & A,B & 4 \\
\hline $\begin{array}{l}\text { Cedrus atlantica (Endl.) } \\
\text { Manetti ex Carrière }\end{array}$ & A,B,C,D & 6 & Pinus mиgo Turra.'Mughus' & A & 6 \\
\hline $\begin{array}{l}\text { Cedrus atlantica (Endl.) } \\
\text { Manetti ex Carrière } \\
\text { 'Glauca' }\end{array}$ & A,B,D & 6 & Pinus nigra J. F. Arnold & $\mathrm{A}, \mathrm{C}$ & 6 \\
\hline $\begin{array}{l}\text { Cedrus atlantica (Endl.) } \\
\text { Manetti ex Carrière } \\
\text { 'Glauca Pendula' }\end{array}$ & $\mathrm{A}, \mathrm{B}$ & 6 & $\begin{array}{l}\text { Pinus nigra J. F. Arnold } \\
\text { 'Austriaca' }\end{array}$ & $\mathrm{D}$ & 6 \\
\hline $\begin{array}{l}\text { Cedrus atlantica (Endl.) } \\
\text { Manetti ex Carrière } \\
\text { 'Glauca Pyramidalis' }\end{array}$ & B & 6 & Pinus pinea $\mathrm{L}$. & A,C,D & 6 \\
\hline $\begin{array}{l}\text { Cedrus atlantica (Endl.) } \\
\text { Manetti ex Carrière } \\
\text { 'Pendula' }\end{array}$ & $\mathrm{A}, \mathrm{B}$ & 6 & Pinus strobus L. & $\mathrm{D}$ & 6 \\
\hline $\begin{array}{l}\text { Cedrus deodara (Roxb. } \\
\text { ex D.Don) G.Don }\end{array}$ & $\mathrm{B}, \mathrm{D}$ & 6 & Pinus sylvestris L. & $\mathrm{D}$ & 6 \\
\hline $\begin{array}{l}\text { Cedrus deodara (Roxb. } \\
\text { ex D.Don) G.Don } \\
\text { 'Aurea' }\end{array}$ & $\mathrm{B}, \mathrm{D}$ & 6 & Pinus wallichiana A.B.Jacks. & A,B & 6 \\
\hline $\begin{array}{l}\text { Cedrus deodara (Roxb. } \\
\text { ex D.Don) G.Don } \\
\text { 'Feelin Blue' }\end{array}$ & A & 6 & $\begin{array}{l}\text { Armeria maritima (Mill.) } \\
\text { Willd. 'Splendens' }\end{array}$ & A & 6 \\
\hline $\begin{array}{l}\text { Cedrus deodara (Roxb. } \\
\text { ex D.Don) G.Don } \\
\text { 'Golden Horizon' }\end{array}$ & A & 6 & Arundo donax L.'Variegata' & $\mathrm{B}$ & 6 \\
\hline $\begin{array}{l}\text { Cedrus deodara (Roxb. } \\
\text { ex D.Don) } \\
\text { G.Don'Pendula' }\end{array}$ & $\mathrm{B}$ & 6 & Festuca glauca Vill. & A & 6 \\
\hline
\end{tabular}

*City Park: A-Hüdavendigar, B-Soganli Botanical, C-Resat Oyal, D-Merinos 
Table 1. Continuing

\begin{tabular}{|c|c|c|c|c|c|}
\hline Taxons & City Park* & $\begin{array}{l}\text { Toxic } \\
\text { Groups }\end{array}$ & Taxon & $\begin{array}{l}\text { City } \\
\text { Park* }\end{array}$ & $\begin{array}{l}\text { Toxic } \\
\text { Groups }\end{array}$ \\
\hline Cedrus libani A. Rich. & $\mathrm{B}, \mathrm{C}$ & 6 & $\begin{array}{l}\text { Imperata cylindrica (L.) } \\
\text { Raeusch. 'Red Baron' }\end{array}$ & $\mathrm{A}$ & 6 \\
\hline Picea alba Link. & $\mathrm{B}$ & 6 & $\begin{array}{l}\text { Miscanthus sinensis } \\
\text { Andersson 'Variegatus' }\end{array}$ & A & 6 \\
\hline $\begin{array}{l}\text { Picea excelsa (Lam.) } \\
\text { Link }\end{array}$ & $\mathrm{B}, \mathrm{D}$ & 6 & $\begin{array}{l}\text { Phalaris arundinacea L. } \\
\text { 'Picta' }\end{array}$ & A & 6 \\
\hline $\begin{array}{l}\text { Picea excelsa (Lam.) } \\
\text { Link 'Inversa' }\end{array}$ & B & 6 & $\begin{array}{l}\text { Phyllostachys } \\
\text { viridiglaucescens (Carrière) } \\
\text { Rivière \& C.Rivière }\end{array}$ & $\mathrm{B}, \mathrm{C}$ & 6 \\
\hline $\begin{array}{l}\text { Picea glauca } \\
\text { Moench.'Conica' }\end{array}$ & $\mathrm{B}, \mathrm{D}$ & 6 & Stipa tenuifolia Steud. & A & 6 \\
\hline $\begin{array}{l}\text { Picea orientalis }(\mathrm{L} .) \\
\text { Link. }\end{array}$ & $\mathrm{C}, \mathrm{D}$ & 4 & Portulaca sp. & $\mathrm{A}, \mathrm{B}, \mathrm{C}$ & $2,3,5$ \\
\hline Picea pungens Engelm. & $\mathrm{D}$ & 4 & Primula sp. & $\mathrm{C}$ & 6 \\
\hline $\begin{array}{l}\text { Picea pungens } \\
\text { Engelm.'Glauca' }\end{array}$ & B & 4 & Punica granatum $\mathrm{L}$. & $\mathrm{B}, \mathrm{C}$ & 2 \\
\hline $\begin{array}{l}\text { Picea pungens } \\
\text { Engelm.'Glauca } \\
\text { Globosa' }\end{array}$ & B & 4 & Clematis sp. & $\mathrm{D}$ & $2,4,5$ \\
\hline $\begin{array}{l}\text { Picea pungens } \\
\text { Engelm.'Glauca } \\
\text { Globosa Nana' }\end{array}$ & A & 4 & $\begin{array}{l}\text { Chaenomeles japonica } \\
\text { (Thunb.) Lindl. ex Spach }\end{array}$ & $\mathrm{C}$ & 6 \\
\hline $\begin{array}{l}\text { Cotoneaster dammeri } \\
\text { C.K.Schneid. }\end{array}$ & $\mathrm{C}$ & 6 & Sorbus aucuparia L. & B & 6 \\
\hline $\begin{array}{l}\text { Cotoneaster franchetii } \\
\text { Bois }\end{array}$ & $\mathrm{B}, \mathrm{C}, \mathrm{D}$ & 2,5 & Spiraea .sp. & $\mathrm{B}, \mathrm{D}$ & 6 \\
\hline $\begin{array}{l}\text { Cotoneaster } \\
\text { horizontalis Decne. }\end{array}$ & $\mathrm{B}, \mathrm{D}$ & 6 & Populus alba $\mathrm{L}$. & $\mathrm{C}$ & 6 \\
\hline $\begin{array}{l}\text { Crataegus oxyacantha } \\
\text { L. }\end{array}$ & B & 6 & Populus nigra $L$. & $\mathrm{C}$ & 6 \\
\hline $\begin{array}{l}\text { Eriobotrya japonica } \\
\text { (Thunb.) Lindl. }\end{array}$ & $\mathrm{C}$ & 1,5 & Salix babylonica L. & A,B,C,D & 6 \\
\hline $\begin{array}{l}\text { Laurocerasus } \\
\text { officinalis M.Roem. }\end{array}$ & $\mathrm{C}, \mathrm{D}$ & 1,5 & Salix caprea L. & $\mathrm{A}, \mathrm{B}$ & 6 \\
\hline $\begin{array}{l}\text { Laurocerasus } \\
\text { officinalis M.Roem. } \\
\text { 'Otto Luyken' }\end{array}$ & $\mathrm{C}$ & 1,5 & Salix matsudana Koidz. & B & 6 \\
\hline $\begin{array}{l}\text { Malus floribunda } \\
\text { Siebold ex Van Houtte }\end{array}$ & A,B,C,D & 1,5 & Salix nigra Marshall & $\mathrm{C}$ & 6 \\
\hline $\begin{array}{l}\text { Malus x purpurea } \\
\text { (Barbier.) Rehd. 'Eleyi' }\end{array}$ & B & 1,5 & $\begin{array}{l}\text { Koelreuteria paniculata } \\
\text { Laxm. }\end{array}$ & $\mathrm{C}$ & 6 \\
\hline $\begin{array}{l}\text { Photinia serrulata } \\
\text { Siebold \& Zucc. }\end{array}$ & A,B,C,D & 6 & $\begin{array}{l}\text { Ailanthus altissima (Mill.) } \\
\text { Swingle }\end{array}$ & $\mathrm{C}$ & 2,4 \\
\hline $\begin{array}{l}\text { Prunus cerasifera Ehrh. } \\
\text { 'Atropurpurea' }\end{array}$ & $\mathrm{C}$ & 1,5 & Petunia sp. & A & 6 \\
\hline $\begin{array}{l}\text { Prunus cerasifera Ehrh. } \\
\text { 'Pissardii Nigra' }\end{array}$ & $\mathrm{A}, \mathrm{B}, \mathrm{D}$ & 1,5 & Tamarix hispida Willd. & $\mathrm{D}$ & 6 \\
\hline $\begin{array}{l}\text { Prunus persica (L.) } \\
\text { Batsch }\end{array}$ & $\mathrm{C}$ & 1,5 & Tamarix gallica $\mathrm{L}$. & B & 6 \\
\hline $\begin{array}{l}\text { Prunus persica (L.) } \\
\text { Batsch 'Cardinal' }\end{array}$ & B & 1,5 & Taxus baccata $\mathrm{L}$. & $\mathrm{A}, \mathrm{B}, \mathrm{C}, \mathrm{D}$ & 1,5 \\
\hline $\begin{array}{l}\text { Prunus serrulata } \\
\text { Lindl.'Kanzan' }\end{array}$ & $\mathrm{A}, \mathrm{B}$ & 1,5 & $\begin{array}{l}\text { Taxus baccata L.'Fastigiata } \\
\text { Aurea' }\end{array}$ & B & 1,5 \\
\hline $\begin{array}{l}\text { Prunus serrulata Lindl. } \\
\text { 'Kiku Shidore Zakura' }\end{array}$ & A & 1,5 & $\begin{array}{l}\text { Taxus baccata (Parsons) } \\
\text { Rehder 'Repandens' }\end{array}$ & B & 1,5 \\
\hline
\end{tabular}

*City Park: A-Hüdavendigar, B-Soganli Botanical, C-Resat Oyal, D-Merinos 
Table 1. Continuing

\begin{tabular}{|c|c|c|c|c|c|}
\hline Taxons & City Park* & $\begin{array}{l}\text { Toxic } \\
\text { Groups }\end{array}$ & Taxon & $\begin{array}{l}\text { City } \\
\text { Park* }\end{array}$ & $\begin{array}{l}\text { Toxic } \\
\text { Groups }\end{array}$ \\
\hline $\begin{array}{l}\text { Prunus serrulata Lindl. } \\
\text { 'Amanogawa' }\end{array}$ & $\mathrm{B}$ & 1,5 & Camellia japonica L. & $\mathrm{B}, \mathrm{D}$ & 6 \\
\hline $\begin{array}{l}\text { Prunus x yedoensis } \\
\text { Matsum. }\end{array}$ & $\mathrm{C}$ & 1,5 & $\begin{array}{l}\text { Camellia japonica } \\
\text { L.'Adolphe Audusson' }\end{array}$ & A & 6 \\
\hline $\begin{array}{l}\text { Pyracantha coccinea } \\
\text { M.Roem. }\end{array}$ & $\mathrm{B}, \mathrm{D}$ & $2,4,5$ & $\begin{array}{l}\text { Camellia japonica } \\
\text { L.'Debbie' }\end{array}$ & A & 6 \\
\hline Rosa sp. & A,C,D & 6 & Celtis australis L. & $\mathrm{B}, \mathrm{D}$ & 6 \\
\hline Rubus fruticosus L. & $\mathrm{D}$ & 6 & Ulmus laevis Pall. & $\mathrm{C}$ & 6 \\
\hline $\begin{array}{l}\text { Parthenocissus } \\
\text { quinquefolia (L.) } \\
\text { Planch. }\end{array}$ & $\mathrm{C}$ & $3,4,5$ & Ulmus minör Mill. & A & 6 \\
\hline Vitis vinifera $\mathrm{L}$. & $\mathrm{C}$ & 5 & Viola tricolor $\mathrm{L}$. & $\mathrm{D}$ & 6 \\
\hline
\end{tabular}

*City Park: A-Hüdavendigar, B-Soganli Botanical, C-Resat Oyal, D-Merinos
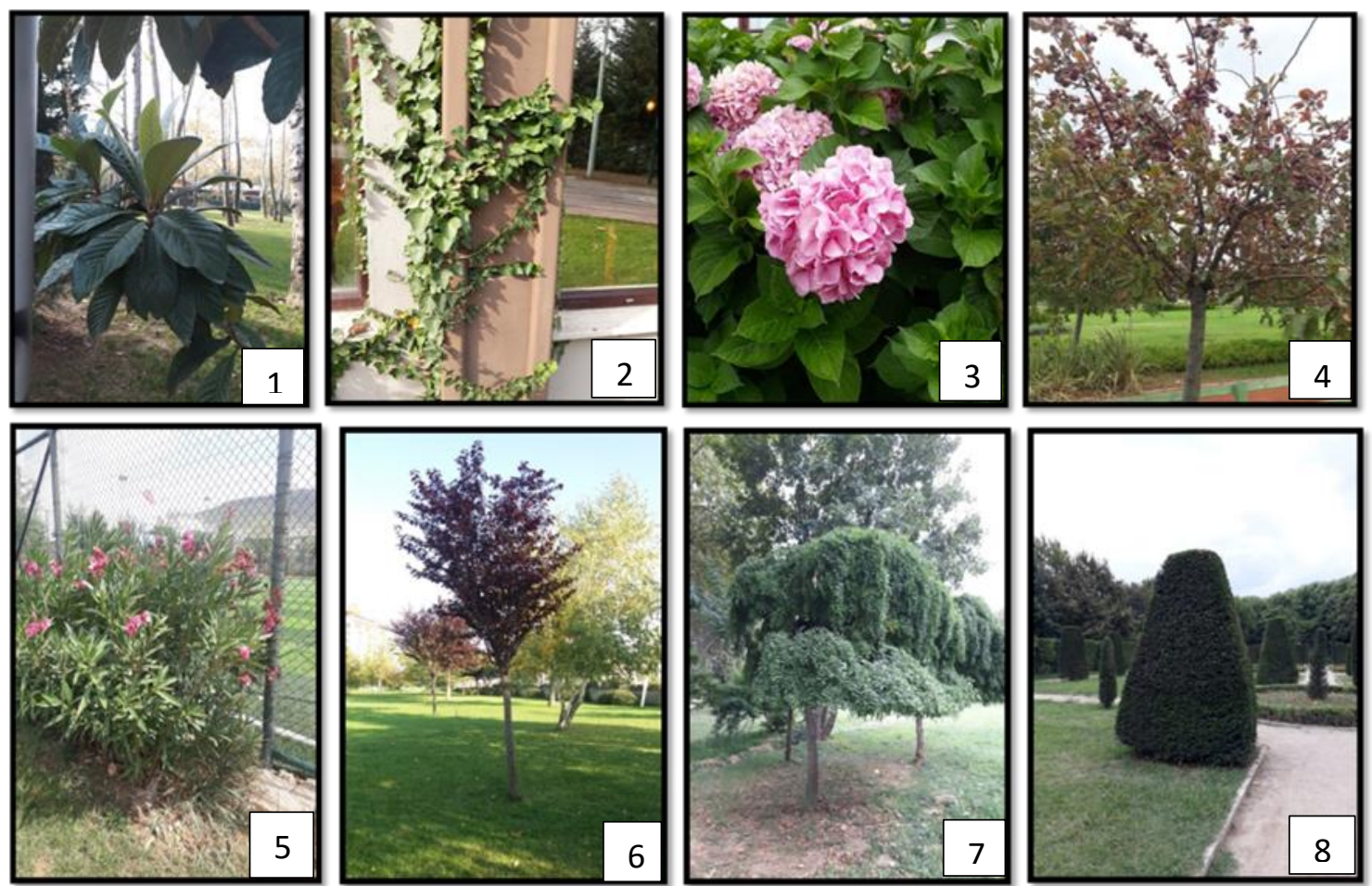

Figure 2. Some of the plants which is in the 1.group (major) toxicity (1-Eriobotrya japonica (Thunb.) Lindl., 2Hedera helix L., 3-Hydrangea macrophylla (Thunb.) Ser., 4-Malus floribunda Siebold ex Van Houtte, 5-Nerium oleander L., 6-Prunus cerasifera Ehrh., 7-Sophora japonica L. 'Pendula',8- Taxus baccata L.) 

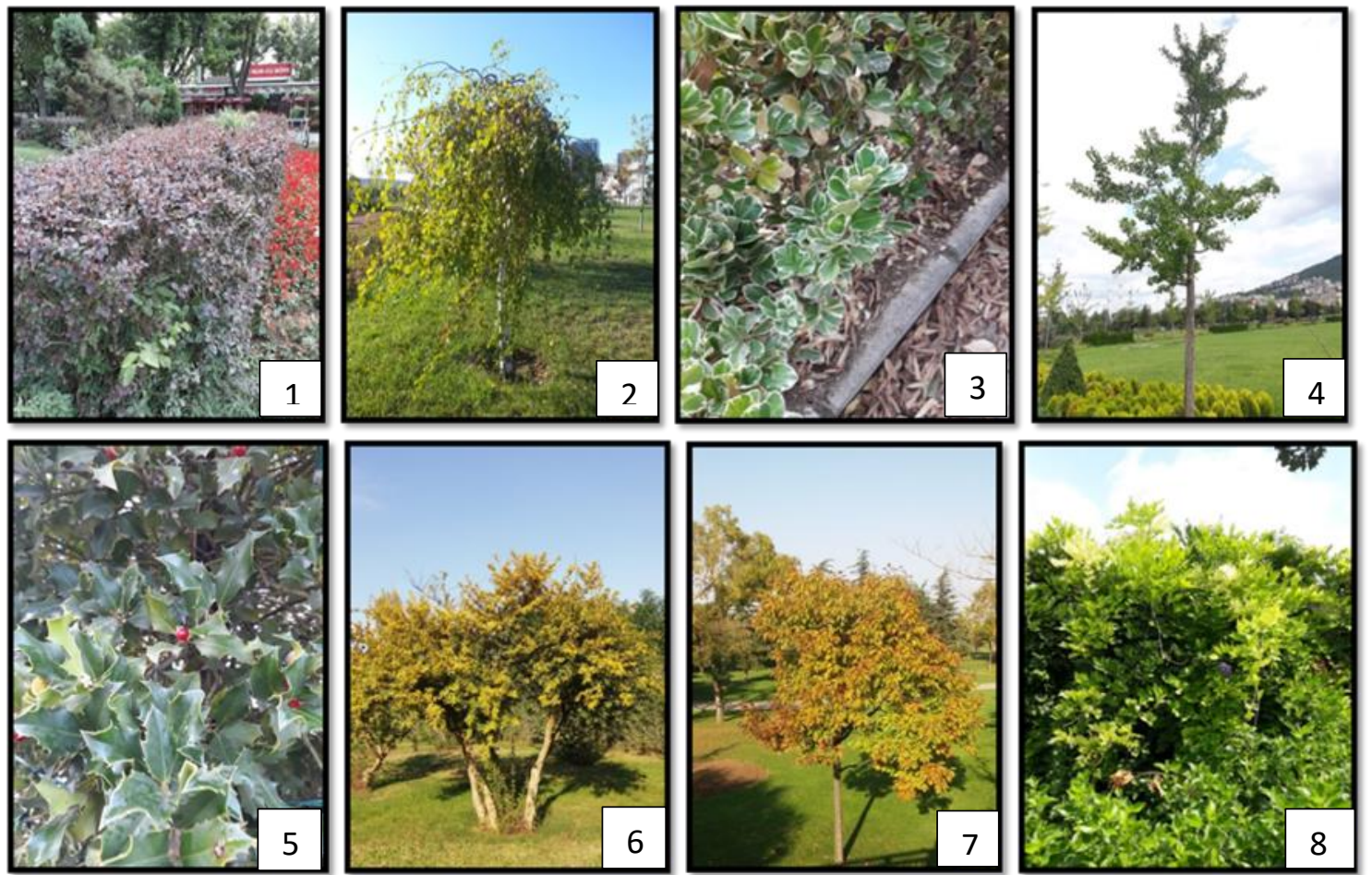

Figure 3. Some of the plants which is in the 2.group (minor) toxicity (1-Berberis thunbergii L. 'Atropurpurea', 2Betula pendula Roth. , 3-Euonymus japonicus L. 'Argentea Variegata', 4-Ginkgo biloba L. , 5-Ilex aquifolium L, 6-Punica granatum L., 7-Quercus rubra L., 8-Wisteria sinensis (Sims) Sweet)
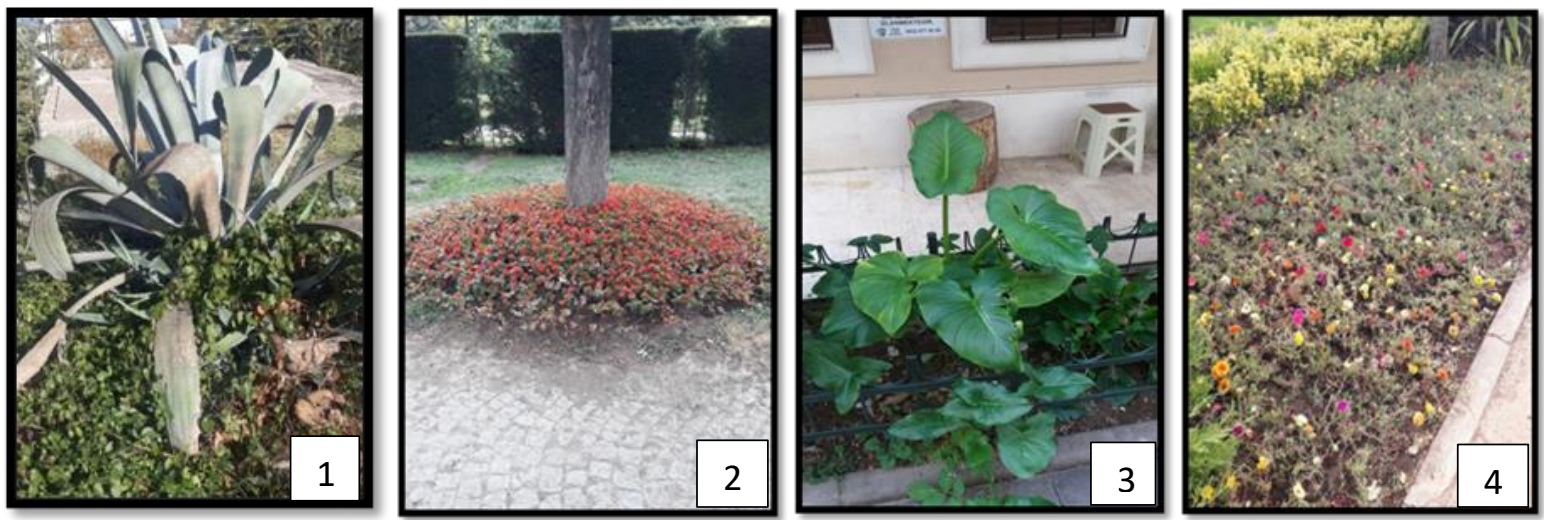

Figure 4. The plants which is in the 3.group (containing oxalate crystals) toxicity (1-Agave americana. L., 2Begonia sp. L., 3-Colocasia sp. Link., 4-Portulaca sp. L.) 

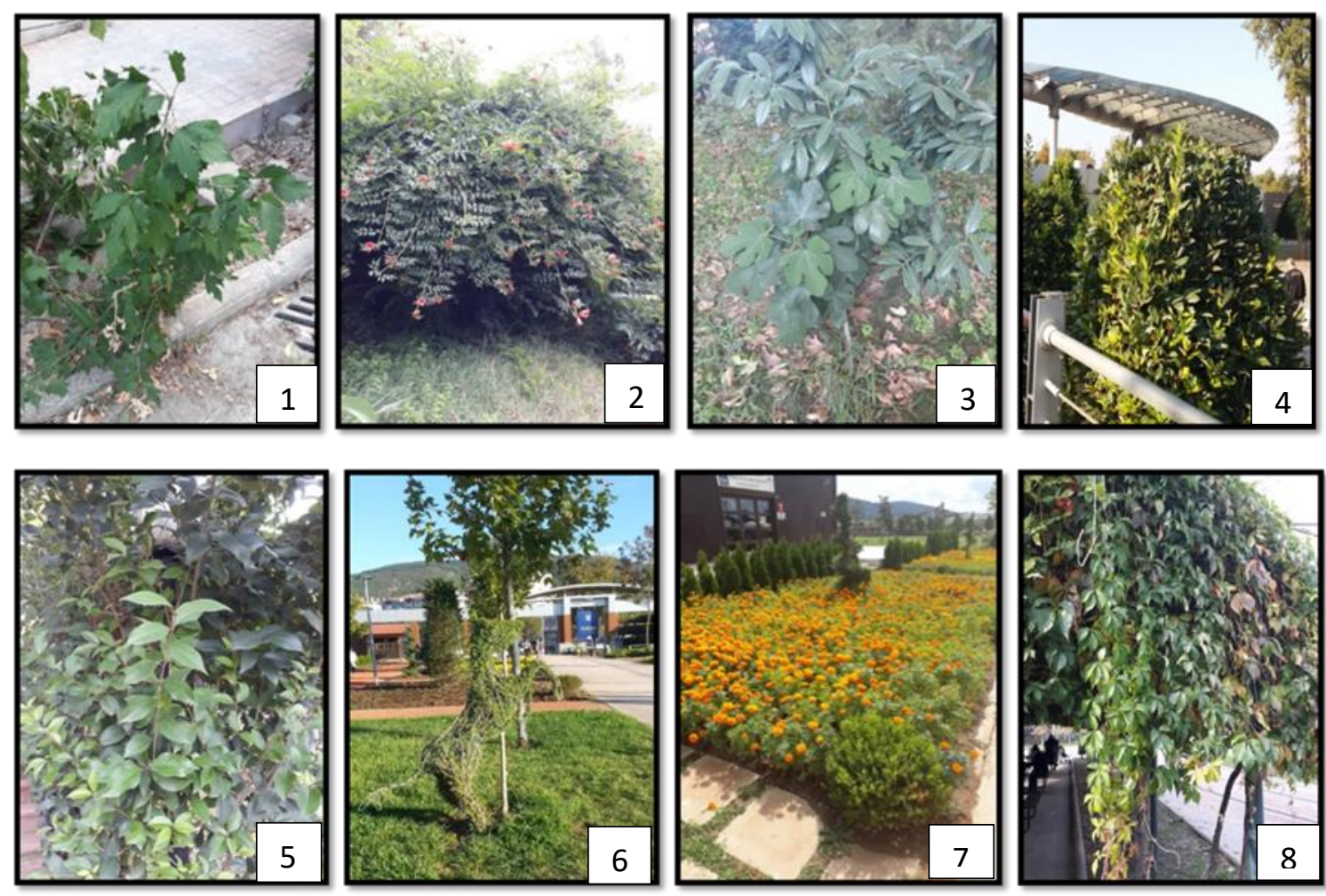

Figure 5. Some of the plants which is in the 4.group (causing dermatitis) toxicity (1-Alcea rosea L., 2-Campsis radicans (L.) Seem, 3-Ficus carica L., 4-Laurus nobilis L., 5-Ligustrum vulgare L., 6-Ligustrum ionandrum Diels, 7-Tagetes erecta L., 8-Parthenocissus quinquefolia (L.) Planch)
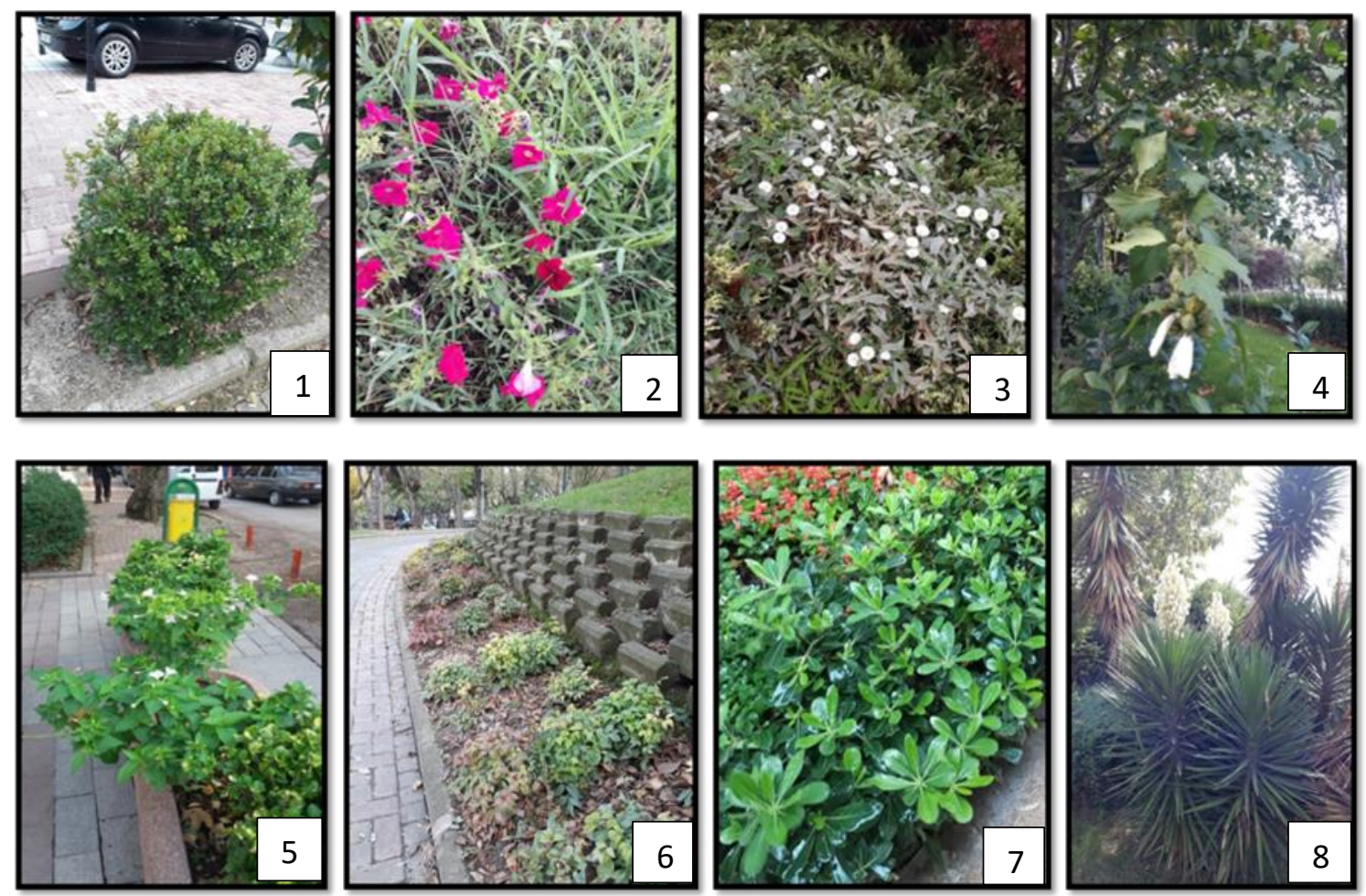

Figure 6. Some of the plants which is in the 5.group (causing animal toxicity) (1-Buxus sempervirens L., 2Clematis sp. L., 3-Convolvulus arvensis L., 4-Hibiscus syriacus L., 5-Mirabilis jalapa L., 6-Nandina domestica Thunb. 'Firepower', 7-Pittosporum tobira (Thunb.) W.T.Aiton 'Nana', 8-Yucca filamentosa L.) 


\subsection{The evaluation of taxa according to the toxic groups}

The evaluations made have shown that taxa detected in city parks are in one or more toxic groups (Table 2), and evaluations according to toxic groups were made taking this feature into consideration. According to the results of the one-way analysis of variance, the total number of taxa in toxic groups and the number of toxic taxa were found to be significant at $\mathrm{p}<0.01$. It has been determined that the most taxa and the most toxic taxa in the six toxic groups are in Soganli Botanical Park, and the least taxa and the least toxic taxa are in Merinos City Park. On the other hand, although the number of taxa in the toxic groups in Reşat Oyal Culture Park and Hüdavendigar City Park is high, it has been determined that the number of toxic taxa is relatively low (Table 3 ).

Table 2.The distribution of taxa in city parks according to toxic groups (Number)

\begin{tabular}{lcccc}
\hline & $\begin{array}{c}\text { Hüdavendigar } \\
\text { City Park }\end{array}$ & $\begin{array}{c}\text { Soganli } \\
\text { Botanical Park }\end{array}$ & $\begin{array}{c}\text { Resat Oyal } \\
\text { Culture Park }\end{array}$ & $\begin{array}{c}\text { Merinos City } \\
\text { Park }\end{array}$ \\
\hline Group1:Major & 9 & 15 & 15 & 8 \\
Toxicity & & & & \\
Group 2:Minor & 23 & 33 & 23 & 13 \\
Toxicity & 1 & 3 & 3 & 1 \\
Group 3:Oxalates & 24 & 29 & 19 & 14 \\
Group 4:Dermatitis & 23 & 43 & 35 & 21 \\
Group 5:Animal & 68 & 97 & 55 & 79 \\
Toxicity & 108 & 159 & 105 & \\
Group 6:Non-Toxic & & & & \\
Total Taxon Number & & & & \\
& & & & \\
\hline
\end{tabular}

Table 3. The number of taxa and toxic taxa in toxic groups

\begin{tabular}{lcccc}
\hline \multicolumn{1}{c}{ City Parks } & $\begin{array}{c}\text { Number } \\
\text { of } \\
\text { Taxon }\end{array}$ & Sig. & $\begin{array}{c}\text { Number of Toxic } \\
\text { Taxon }\end{array}$ & Sig. \\
\hline Resat Oyal Culture Park & $150,00 \mathrm{~b}$ & & $95,00 \mathrm{~b}$ & $0,000^{* * *}$ \\
Soganli Botanical Park & $220,00 \mathrm{a}$ & $0,000^{* * *}$ & $123,00 \mathrm{a}$ & \\
Merinos City Park & $106,00 \mathrm{~d}$ & & $57,00 \mathrm{~d}$ & $80,00 \mathrm{c}$ \\
Hüdavendigar City Park & $148,00 \mathrm{c}$ & & 900 \\
\hline
\end{tabular}

According to the correlation analysis performed in order to determine the relationship between the toxic taxa and the taxa in the city parks within the determined six toxic groups, the correlation coefficient (r) value (+) was found to be positive and a linear relationship was found between the number of taxa and toxic taxa. It has been determined that the number of toxic taxa has increased due to the increase in the number of taxa in six groups in urban parks (Table 4, Figure 2).

Table 4. The correlation analysis between total taxa in toxic groups and toxic taxa

\begin{tabular}{lll}
\hline \multirow{3}{*}{ Total Taxon } & \multicolumn{2}{l}{ Toxic Taxons } \\
\cline { 2 - 3 } & Pearson Correlation (r) & 0,974 \\
& Significant (Sig.) & $0,026^{*}$ \\
\hline
\end{tabular}

**. Correlation is significant at the 0.01 level (2-tailed). *. Correlation is significant at the 0.05 level (2-tailed). 




Figure 7. The relationship between taxa in toxic groups and toxic taxa

According to the results of the correlation analysis performed to determine the relationships between the toxic groups and the number of taxa in the toxic groups, the correlation coefficient $(r)$ value $(+)$ between the total number of taxa in toxic groups and the group of minor toxicity and non-toxic taxa was determined to be positive and a linear relationship has been found to be.

With the increase in the number of taxa, the number of taxa in the minor toxicity and non-toxic group has also increased. However, no relationship was found between the number of taxa that being in the group of high toxicity, oxalates, dermatitis and animal toxicity and the increase in the number of taxa (Table 5, Figure 3).

Table 5. The correlation analysis between toxic groups and taxon numbers

\begin{tabular}{llllllll}
\hline Correlations & $\begin{array}{l}\text { Major } \\
\text { Toxicity }\end{array}$ & $\begin{array}{l}\text { Minor } \\
\text { Toxicity }\end{array}$ & Oxalates & Dermatitis & $\begin{array}{l}\text { Animal } \\
\text { Toxicity }\end{array}$ & Non-Toxic \\
\hline $\begin{array}{l}\text { Total } \\
\text { Taxon }\end{array}$ & $\begin{array}{l}\text { Pearson } \\
\begin{array}{l}\text { Correlation } \\
\text { (r) }\end{array}\end{array}$ & 0,744 & 0,985 & 0,709 & 0,929 & 0,904 & 0,953 \\
& $\begin{array}{l}\text { Significant } \\
\text { (Sig.) }\end{array}$ & 0,256 & $0,015^{* *}$ & 0,291 & 0,071 & 0,096 & $0,047^{*}$ \\
\hline
\end{tabular}

\footnotetext{
**. Correlation is significant at the 0.01 level (2-tailed). *. Correlation is significant at the 0.05 level (2-tailed).
} 

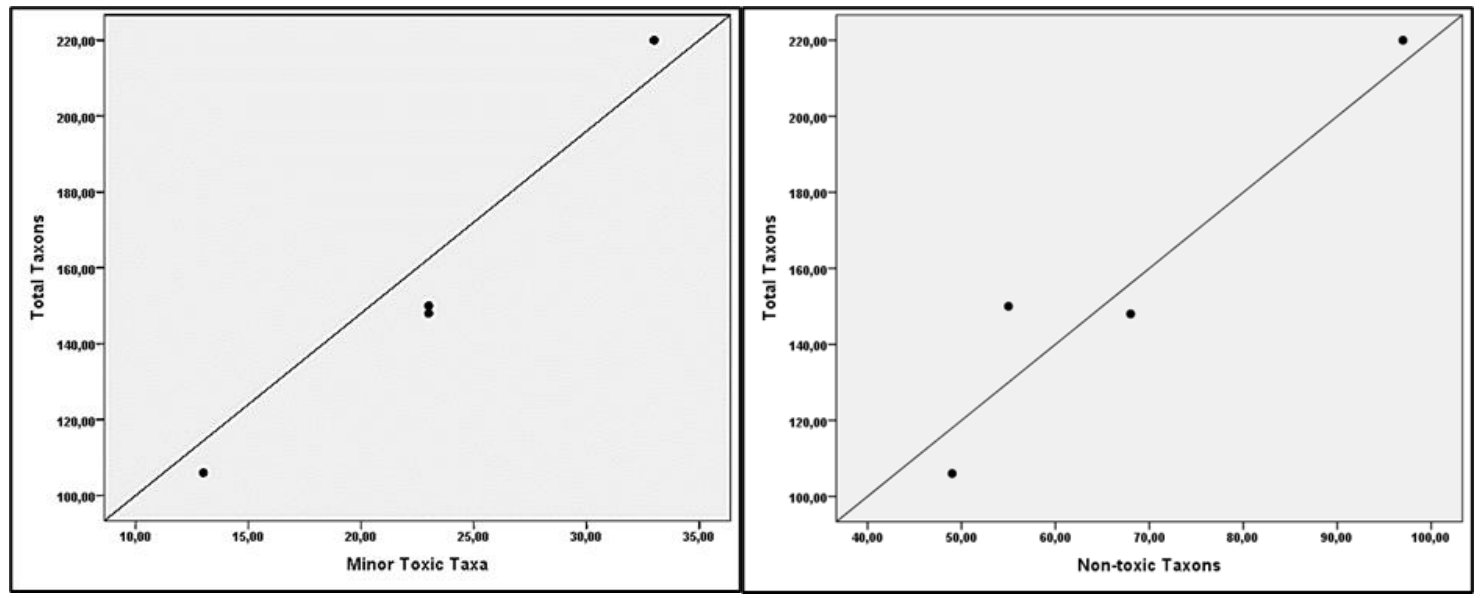

1. Minor Toxicity

2. Non-Toxic

Figure 8 . The relationship between the total number of taxa in the toxic groups and the number of taxa in the minor toxicity and non-toxic groups

According to the two-way analysis of variance between city parks and toxic groups, the interaction of parks, toxic groups and parks $\mathrm{x}$ toxic groups was found to be significant at $\mathrm{p}<0.01$ in terms of taxon numbers (Table $6)$.

Table 6 . The results of the two way analysis of variance between parks and toxic groups

\begin{tabular}{lllll}
\hline Factors & df & Mean Square & F & Sig. \\
\hline Parks & 3 & 1113,458 & 1113,458 & $0,000^{* * *}$ \\
Toxic Groups & 5 & 6077,825 & 6077,825 & $0,000^{* * *}$ \\
Parks x Toxic Groups & 15 & 187,158 & 187,158 & $0,000^{* * *}$ \\
\hline
\end{tabular}

It has been determined the average number of taxa owned by toxic groups in city parks is the lowest in Merinos City Park with 17.66 pieces, to be the highest in Soganli Botanical Park with 36.66 pieces (Table 7).

Table 7. The average number of taxa of toxic groups in city parks

\begin{tabular}{ll}
\hline City Parks & Avarage Number of Taxa of Toxic Groups \\
\hline Resat Oyal Culture Park & $25,00 \mathrm{~b}$ \\
Soganli Botanical Park & $36,66 \mathrm{a}$ \\
Merinos City Park & $17,66 \mathrm{c}$ \\
Hüdavendigar City Park & $24,83 \mathrm{~b}$ \\
\hline
\end{tabular}

*) Mean separation in columns by Duncan's Multiple Range Test, $\mathrm{P}=0.05$.

When the distribution of the taxa in city parks in terms of toxic groups is evaluated; it was determined that the highest number of taxa in all city parks is in Group 6 (non-toxic) with an average of 67.25 pieces, followed by the taxa in Group 5 (animal toxicity) with an average of 30.75 pieces. It was determined that the least average number of taxa is in Group 3 (oxalates) (Table 8). 
Table 8 . The avarage number of taxa in toxic groups

\begin{tabular}{ll}
\hline Toxic Groups & Average Taxon Number \\
\hline Group1:Major Toxicity & $11,75 \mathrm{e}^{*}$ \\
Group2:Minor Toxicity & $23,00 \mathrm{c}$ \\
Group3:Oxalates & $2,00 \mathrm{f}$ \\
Group4:Dermatitis & $21,50 \mathrm{~d}$ \\
Group5:Animal Toxicity & $30,75 \mathrm{~b}$ \\
Group6:Non-toxic & $67,25 \mathrm{a}$ \\
\hline
\end{tabular}

*) Mean separation in columns by Duncan's Multiple Range Test, $\mathrm{P}=0.05$.

When the interaction of parks $\mathrm{x}$ toxic groups, which is important at the level of $\mathrm{p} \leq 0.01$, is evaluated; It was observed that the average number of taxa in group 1 was highest in Reşat Oyal Culture Park and Soğanlı Botanic Park, and the number of taxa in Group 2, 3, 4, 5 and 6 was highest in Soganli Botanical Park (Table 9).

Table 9. City parks $\mathrm{x}$ toxic groups interaction average number of taxa

\begin{tabular}{|c|c|c|}
\hline City Parks & Toxic Groups & Average Taxon Number \\
\hline \multirow[t]{6}{*}{ Resat Oyal Culture Park } & Group1:Major Toxicity & $15,00 \mathrm{j}^{*}$ \\
\hline & Group2:Minor Toxicity & $23,00 \mathrm{hi}$ \\
\hline & Group3:Oxalates & $3,00 \quad 1$ \\
\hline & Group4:Dermatitis & $19,00 \mathrm{ij}$ \\
\hline & Group5:Animal Toxicity & $35,00 \mathrm{f}$ \\
\hline & Group6:Non-toxic & $55,00 \mathrm{c}$ \\
\hline \multirow[t]{6}{*}{ Soganli Botanical Park } & Group1:Major Toxicity & $15,00 \mathrm{j}$ \\
\hline & Group2:Minor Toxicity & $33,00 \mathrm{fg}$ \\
\hline & Group3:Oxalates & $3,00 \quad 1$ \\
\hline & Group4:Dermatitis & $29,00 \mathrm{~g}$ \\
\hline & Group5:Animal Toxicity & $43,00 \mathrm{e}$ \\
\hline & Group6:Non-toxic & 97,00 a \\
\hline \multirow[t]{6}{*}{ Merinos City Park } & Group1:Major Toxicity & $8,00 \quad \mathrm{k}$ \\
\hline & Group2:Minor Toxicity & $13,00 \mathrm{j}$ \\
\hline & Group3:Oxalates & $1,00 \quad 1$ \\
\hline & Group4:Dermatitis & $14,00 \mathrm{j}$ \\
\hline & Group5:Animal Toxicity & $21,00 \mathrm{i}$ \\
\hline & Group6:Non-toxic & $49,00 \mathrm{~d}$ \\
\hline \multirow[t]{6}{*}{ Hüdavendigar City Park } & Group1:Major Toxicity & $9,00 \quad \mathrm{k}$ \\
\hline & Group2:Minor Toxicity & 23,00 hi \\
\hline & Group3:Oxalates & $1,00 \quad 1$ \\
\hline & Group4:Dermatitis & $24,00 \mathrm{~h}$ \\
\hline & Group5:Animal Toxicity & $24,00 \mathrm{~h}$ \\
\hline & Group6:Non-toxic & $68,00 \quad b$ \\
\hline
\end{tabular}

*) Mean separation in columns by Duncan's Multiple Range Test, $\mathrm{P}=0.01$.

\section{Discussion and Conclusion}

This study aims to determine the toxic effects of design (landscape) plants used in city parks on humans and animals and to classify them according to their toxicity groups. And it has been determined that there are 299 taxa in 4 city parks in Bursa province. These taxa belong to 69 families and are 190 species and 109 cultivars. It was determined that taxa can being more than one toxicity group. It was observed that $10.07 \%$ of these taxa are 
in the major toxicity group, $21.07 \%$ of these taxa are in the minor toxicity group, $1.67 \%$ of these taxa are in the containing oxalate crystals group, $20.07 \%$ of these taxa are in the causing dermatitis, and $26.08 \%$ of these taxa are in the causing animal toxicity group. The rate of taxa in the non-toxic group was determined to be $20.40 \%$.

It was determined that $56.76 \%$ of the design plants in all city parks have toxic properties, the highest rate of toxic design plants is in Reşat Oyal Culture Park with $63.33 \%$ and the lowest toxic plants are in Merinos City Park with $53.77 \%$.

Some plants used in the treatment of many diseases can cause toxic effects on humans and animals due to the components they contain (Y1lmaz et al. 2006). The studies have confirmed that species belonging to the same genus may have different toxic properties and also that toxic compounds may differ from species to species. Indeed, A. pseudoplatanus, A. rubrum, A. saccharinum and A. saccharum species belonging to the genus Acer were found to have toxic properties. However, A. campestre, A. ginnala, A. negundo, A. palmatum and A. platanoides species were found to haven't toxic properties. This situation was expressed in a similar way in the study conducted by Yilmaz et al. (2006).

As a result of the evaluations, it was determined that the design plants in city parks, as stated by Filmer (2012), include that major toxic compounds such as alkaloids, glycosides, oxalate, saponin, tannin, essential oil, fixed oil, bitter substance, terpene, pectin, resinous compounds, allergic and antigenic proteins, phenol, flavone, terpenoid etc.

On the other hand, it was observed that the taxa containing oxalate crystals were mostly found in Soganli Botanical Park and Resat Oyal Culture Park. The taxa that causing dermatitis and the taxa that causing animal toxicity were mostly found in Soganli Botanical Park.

It has been determined that the taxa causing animal toxicity are in 27 families and the family with the most taxa is the Rosaceae family, followed by the Fagaceae, Cupressaceae and Fabaceae families, respectively. Y1lmaz (1990), determined that taxa belonging to 24 families which causing animal toxicity in his study named "Toxicological Properties of Important Poisonous Plants Growing in Bursa Region". And he stated that the plants that causing animal toxicity are mostly in the Fabaceae family.

In the study carried out by Altıntaş (1995); Nerium oleander, Aesculus hippocastanum, Euonymus japonicus, Ligustrum vulgare, Ligustrum japonicum and Robinia pseudoacacia are the most commonly used toxic ornamental plants in Istanbul parks and gardens. It has been observed that these plants, which are named as toxic plants, are also used in Bursa City Parks, especially Nerium oleander species, which is in the group of major toxicity are found in a large number of city parks and Robinia pseudoacacia species, which is in the group of major toxicity, are found in a large number of city parks.

Consequently; Some of design (landscape) plants used for aesthetic and functional purposes in landscape design works may have negative effects on humans and animals due to their toxic components reveal that the designer needs more information about the characteristics of the used plants. Thus, it will be ensured that the existing toxicity is the least dangerous with the designs considering the toxic compounds of the plants, the toxicity of the plants, the degree of poison and the toxic organs. In addition, placing plant identification plates containing descriptive and cautionary information about plants in city parks, which are used by a vast majority of public, will contribute to raising awareness and minimizing the effects of toxic components in plants.

By prohibiting the use of plants with major toxic effects in landscape design projects approved by municipalities, the use of these plants can be seriously reduced.

In addition to these, a data matrix system can be developed for plants in today's rapidly advancing technology. Thanks to this system, the characteristics and toxicity status of the plant can be learned from the database on the internet. In this way, children's interest in botanical science can be increased.

\section{Acknowledgements}

This study was produced from the Master's Thesis titled “A Research on Toxicological Properties of Bursa City Parks's Design Plants". 


\section{References}

1. Altıntaş, N. (1995). İstanbul Park ve Bahçelerinde Rastlanan Zehirli Süs Bitkileri. Yüksek Lisans Tezi, İÜ, Fen Bilimleri Enstitüsü Orman Mühendisliği Anabilim Dalı, İstanbul.

2. Anonymus (1998). The Hillier Manual of Trees And Shrubs. Pocket edition. A David and Charles Book.

3. Aydın, N. (2010). Edirne'de Yetişen Bazı Zehirli Bitkilerin Yaprak ve Gövdesindeki Kalsiyum Oksalat Kristallerinin Araştırılması. Yüksek Lisans Tezi, TÜ, Fen Bilimleri Enstitüsü Biyoloji Anabilim Dalı, Edirne.

4. Atasoy, N. (2012). Bina İçi Mekânlarda Kullanılan Zehirli Süs Bitkileri Üzerinde Araştırmalar. Doktora Tezi, MÜ, Fen Bilimleri Enstitüsü Biyoloji Anabilim Dalı, İstanbul.

5. Akdeniz, N.S., Ender, E., Zencirkiran, M. (2017). Evaluation of Ecolocigal Tolerance and Requirements of Exotic Conifers in the Urban Landscape of Bursa. Fresenius Environmental Bulletin 26, (10):6064-6070.

6. Baytop, T. (1963). Türkiye'nin Tıbbi ve Zehirli Bitkileri. İstanbul Üniversitesi Tıp Fakültesi Yayınları, No:1039, İstanbul.

7. Davis, P. H. (1965-1988). Flora of Turkey and The East Aegean Islands. Edinburgh University Press. Volume 1-10. Edinburgh.

8. Dirr, M. A. (1992). Manual of Woody Landscape Plants: Their Identification, Ornamental Characteristics, Culture Propagation and Uses. Varsity Press.

9. DiTomaso, J. M. (2019). List of Plants Reported to be Poisonous to Animals in the United States. Davis Weed Research and Information Center, United States, Ithaca.

10. Elings, M. (2006). People-Plant Interaction. The physiological, psychological and sociological effects of plants on people. Chapter 4. Jan Hassink and Majken van Dijk (eds.), Farming for Health, 43-55. Springer. Printed in the Netherlands.

11. Filmer, A. K. (2012). Safe and Poisonous Garden Plants. University of California, Davis. USA. 31p.

12. Frumkin, H. (2001). Beyond ToxicityHuman Health and the Natural Environment. American Journal of Preventive Medicine, 20 (3): 234-240.

13. IBM Corp. Released (2013). IBM SPSS Statistics for Windows, Version 22.0. Armonk, NY: IBM Corp.

14. Karaşah, B., Var, M. (2012). Trabzon ve Bazı İlçelerinde Kent Dokusundaki Bitkilendirme Tasarımlarının Ölçü-Form Açısından İrdelenmesi. Bartın Orman Fakültesi Dergisi, 14: 1-11.

15. Knight, A.P. (2007). A Guide to Poisonous House and Garden Plants, Teton Newmedia, Jackson, Wyoming, USA, $421 \mathrm{p}$.

16. Krussman, G. (1984-1986). Manual Of Cultivated Broad-Leaved Trees and Shrubs. Timber Press, Portland, Oregon.

17. Lewis, C.A. (1995). Human Health and Well-Being: The Psychological, Physiological, and Sociological Effects of Plants on People. Acta Horticulturae:391.

18. Lewis S. Nelson, L.S., Shih, R.D., Balick, M.J. (2007). Handbook of Poisonous and Injurious Plants. Botanical Garden, Springer, New York, 347p.

19. Martin,C., Butelli, E., Petroni, K., Tonellib, C. (2011). How Can Research on Plants Contribute to Promoting Human Health?,The Plant Cell, Vol. 23: 1685-1699.

20. Muca, B., Özçelik, Ş., Koca, A., Yıldırım, B. (2012). Isparta's (Turkey) Poisonous Plants Of Public Access Places. Biological Diversity and Conservation, 5(1): 23-30.

21. Polunin, O. (1969). Flowers of Europe. Oxford Univ. Press. London.

22. Polunin, O., Huxley, A. (1981). Flowers of the Mediterranean. Chatto and Windus. London.

23. Robinson, N. (1992). The Planting Design Handbook, Gower Publishing Company Limited Gower House Craft Road Aldershot Hampshire Gu11 3HR, England, 271p.

24. Sarı, D., Karaşah, B. (2018). Bitkilendirme Tasarımı Öğeleri, İlkeleri ve Yaklaşımlarının Peyzaj Tasarımı Uygulamalarında Tercih Edilirliği Üzerine Bir Araştırma. Megaron, 13(3): 470-47.

25. Scarfone, S.C. (2007). Professional Planting Design An Architectural and Horticultural Approach for Creating Mixed Bed Plantings, John Wiley\&Sons. Inc., Hoboken, New Jersey. 272p

26. Wagstaff, D.J. (2008). International Poisonous Plants Checklist An Evidence-Based Reference. CRC Press is an imprint of the Taylor \& Francis Group, United States, 462p.

27. Yaltırık, F. (1993). Dendrology Textbook II. Angiospermae (Angiosperms) Vol. I. Istanbul.

28. Yener, D., Seyidoğlu, N. (2010). Peyzaj Düzenlemelerinde Zehirli Bitkiler. IV. Ulusal Süs Bitkileri Kongresi Bildiri Kitabi, 20 Ekim 2010, Mersin.

29. Yılmaz, O. (1990). Bursa Yöresinde Yetişen Zehirli Bitkilerin Toksikolojik Özellikleri. Doktora Tezi, UÜ. Sağlık Bilimleri Enstitüsü Veteriner Farmakoloji ve Toksikoloji Anabilim Dalı, Bursa.

30. Yılmaz, H., Akpınar, E., Yılmaz, H. (2006). Peyzaj Mimarlı̆̆ Bitkilerinin Toksikolojik Özellikleri. SDÜ Orman Fakültesi Dergisi, A (1) : 82-95. 
31. Zencirkıran, M. (2004). Bursa Kent Peyzajında Kullanılan Bitki Türleri ve Bu Amaç İçin Kullanılabilecek Yerli ve Yabancı Orjinli Bitkilerin Saptanması. Uludağ Üniversitesi Bilimsel Araştırma Projesi No:2002/24, 307s.

32. Zencirkıran, M. (2009). Determination Of Native Woody Landscape Plants In Bursa and Uludag. African Journal of Biotechnology, 8(21): 5737-5746.

33. Zencirkıran, M. (2013). Peyzaj Bitkileri I. (Açık Tohumlu Bitkiler-Gymnospermae). 1. Basım. Nobel Akademik Yayıncılık. Yayın Nu:605, Fen Bilimleri Nu:57, Ankara. 475 s.

34. Zencirkıran, M., Çelik, B.H., Müdük, B., Görür, A., Çetiner, S., Eraslan, E., Tanrıverdi, D. (2018). İç Mekan Tasarım Bitkilerinin Kullanıcılar İçin Toksik Özellikler Bakımından Değerlendirilmesi. Bartın Orman Fakültesi Dergisi, 20(1): 26-31.

35. Zencirkıran, M, Altay Ender, E., Altun, G. (2019). A Research on Attractive Flowered Exotic Woody Landscape Plant Species Used in Urban Green Spaces in Bursa. Chapter 1: 9-26. Researches in Landscape and Ornamental Plants (Edt. Prof.Dr. Murat Zencirkıran). GeceKitaplığı/Gece Publishing, ISBN:978-6257958-27-1, Ankara. 